\title{
DL-3-n-butylphthalide therapy for Parkinson's disease: A randomized controlled trial
}

\author{
HAIYAN ZHOU ${ }^{1 *}$, MING YE ${ }^{2 *}$, WENFANG XU ${ }^{2 *}$, MEILING YU ${ }^{3}$, XIAOLIN LIU ${ }^{2}$ and YUHUA CHEN ${ }^{4}$ \\ Departments of ${ }^{1}$ Gerontology, ${ }^{2}$ Neurology and ${ }^{3}$ Pharmacy, The First Affiliated Hospital of Bengbu Medical College, \\ Bengbu, Anhui 233004; ${ }^{4}$ Department of Neurology, Anhui Provincial Hospital Affiliated to \\ Anhui Medical University, Hefei, Anhui 230001, P.R. China
}

Received January 23, 2018; Accepted December 21, 2018

DOI: $10.3892 /$ etm.2019.7397

\begin{abstract}
Currently available treatments for Parkinson's disease (PD) do not delay or prevent disease development and progression. DL-3-n-butylphthalide (NBP), isolated from Apium graveolens seeds, alleviates oxidative damage and mitochondrial dysfunction. It has been revealed to reduce the loss of dopamine neurons in pre-clinical PD models, and has been approved for the treatment of ischemic stroke patients. The purpose of the present study was to examine whether NBP has the capacity provide a benefit for PD patients and to slow disease progression. A randomized, controlled trial was performed between September 2014 and December 2016. Pairs of patients matched by age, gender and off-medication Unified PD Rating Scale motor subscale (UPDRS-III) scores, were randomly assigned to an NBP treatment group and a control group. All patients continued their originally prescribed medication regimen and those in the NBP group were administered NBP at $200 \mathrm{mg}$ three times daily for 24 weeks. Primary outcome measures were changes in UPDRS-III, including tremor score and non-tremor score, the Pittsburgh sleep quality index (PSQI) and the PD 39-items questionnaire (PDQ) scores. Assessments were completed by blinded evaluators at baseline and 12, 24 and 48 weeks after randomization. All patients were monitored for adverse events (AEs). A total of 103 patients were enrolled in the present study. The NBP group exhibited significantly greater improvements in the non-tremor, PSQI and PDQ-39 scores than the control group, which generally exhibited no improvement. NBP-associated AEs were uncommon and primarily consisted of mild
\end{abstract}

Correspondence to: Mrs. Yuhua Chen, Department of Neurology, Anhui Provincial Hospital Affiliated to Anhui Medical University, 17 Lujiang Road, Hefei, Anhui 230001, P.R. China

E-mail: doctorcyh@163.com

${ }^{*}$ Contributed equally

Key words: DL-3-n-butylphthalide, Parkinson's disease, randomized controlled trial, Unified Parkinson's Disease Rating Scale motor subscale III gastrointestinal symptoms. In conclusion, over the 6-month treatment period, NBP was safe and effective for improving the symptoms and impairing the progression of patients with PD (Trial registry number, ChiCTR1800018892).

\section{Introduction}

Parkinson's disease (PD) is a progressive neurodegenerative disorder that commonly leads to the loss of motor function (akinetic-rigid, tremor and postural instability gait disorder), non-motor symptoms (NMS; sleep disorder and autonomic dysfunction) and cognitive decline (1). Currently available treatments for PD do not delay or prevent disease progression. Strong evidence has emerged that mitochondrial dysfunction, inflammation and increased oxidative stress have pivotal roles in the pathogenesis of PD and these processes provide a scientific basis for testing potential 'neuroprotective' agents.

DL-3-n-butylphthalide (NBP) (Fig. 1) is a synthetic chiral compound developed from L-3-n-butylphthalide that contains the L- and D-isomers of butylphthalide. NBP was initially isolated as a pure component from the seeds of Apium graveolens in 1978 and was first synthesized in 1980 by researchers at the Institute of Medicine of the Chinese Academy of Medical Sciences (2). Pharmacological tests at that time indicated that NBP had significant central nervous system (CNS) effects, including anti-convulsant and sedative activities, but the dose at which NBP had anti-epileptic effects was close to its maximum tolerated dose. The pharmacological effects of NBP on stroke were demonstrated in whole animals, organs, tissues, cells and at the molecular level, and included a significant reduction in cerebral infarction, protection of mitochondrial function and improvement of brain metabolism $(2,3)$. NBP was approved by the State Food and Drug Administration of China as a therapeutic drug for the treatment of ischemic stroke in 2005 based on the results of multicenter phase 2 and 3 randomized controlled clinical trials and was consistently reported to improve neurologic function after stroke, with good safety and tolerability $(4,5)$. NBP has also been reported to prevent oxidative damage and mitochondrial dysfunction, protect against neurotoxicity via an autophagic mechanism in a 1-methyl-4-phenylpyridinium (+)-induced cellular model of PD (6), and protect dopamine neurons in rotenone-induced models of PD (7). Preliminary 
experiments performed in our group indicated that NBP significantly improved dyskinesia, reduced microglial activation, decreased nuclear $\alpha$-synuclein deposition and increased the survival of tyrosine hydroxylase-positive cells in the substantia nigra of the lipopolysaccharide (LPS)-induced PD mouse model. Therefore, it was hypothesized that NBP may have therapeutic efficacy in patients with PD, and the present study was designed to evaluate this.

\section{Materials and methods}

Study design, participants and oversight. The present study was a prospective, single-center, parallel-group, randomized controlled trial. Patients were self-referred or recruited from the First Affiliated Hospital of Bengbu Medical College (Bengbu, China) between September 2014 and December 2016. The final follow-up evaluation was in December 2016. Patients were eligible for participation in the study if they i) had idiopathic PD confirmed by a movement disorder specialist, according to the Parkinson's Disease Society Brain Bank criteria (8); ii) had been receiving a stable medication regimen for a duration of $\geq 6$ weeks or were early PD patients who had no prior medication treatment and were receiving their first consultation; iii) were between 40 and 80 years of age; and iv) had a family member or friend who was willing to also participate. Exclusion criteria included any factor that may have precluded completion of neuropsychological testing; clinically significant gastrointestinal, hepatic, renal, respiratory or cardiovascular system disease; infection; alcoholism; drug addiction; or known hypersensitivity to celery. Patients were evaluated at the Department of Neurology of the First Affiliated Hospital of Bengbu Medical College (Bengbu, China).

Interventions. NBP was selected, as this additional treatment has been used in the clinic for patients with brain infarction and vascular cognitive impairment but no dementia with good safety and tolerability $(2,3)$. In addition, NBP protected dopamine neurons in in vivo and in vitro models of PD. All participants continued their stable medical regimens under the care of their physicians. Patients were matched by age, gender and off-medication Unified Parkinson's Disease Rating Scale motor subscale (UPDRS-III) scores, and randomly assigned at a 1:1 ratio to the NBP group, which received oral NBP $200 \mathrm{mg}$ three times daily based on the dose approved for stroke that is known to exert CNS effects for 24 weeks (5), or the control group. All patients continued their originally prescribed medication regimen during the 48 weeks of the study. Furthermore, in this time, no new PD treatment was provided to the study participants except for the experimental treatment with NBP.

Study procedures. All assessments were performed at the Department of Neurology of the First Affiliated Hospital of Bengbu Medical College (Bengbu, China) after a 12-h overnight withdrawal of dopaminergic medication. Off-medication assessments were completed in the morning (9). Motor signs were evaluated using the UPDRS-III and, based on the predominant motor features in the UPDRS III motor examination section score, the scores were subtyped into tremor scores and non-tremor (bradykinesia plus rigidity) scores. Tremor scores were derived from the sum of UPDRS III items 20 (tremor at

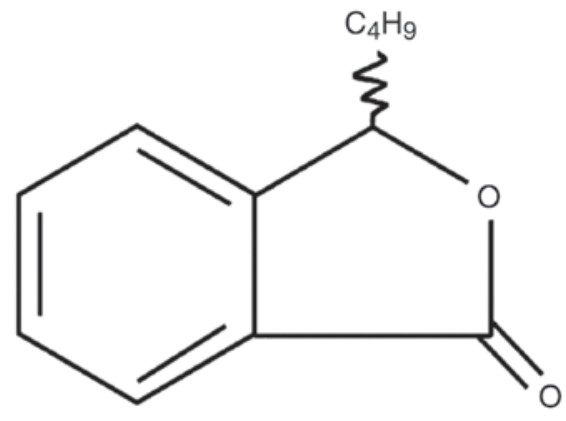

Figure 1. Chemical structure of DL-3-n-butylphthalide, a novel synthetic chiral compound containing the L- and D-isomers of butylphthalide.

rest) and 21 (action or postural tremor of hands). Non-tremor scores were derived from the sum of UPDRS III items 18 (speech), 19 (facial expression), 22 (rigidity), 23 (finger taps), 24 (hand movement), 25 (diadochokinesia), 26 (leg flexibility), 27 (arising from the chair), 28 (posture), 29 (gait), 30 (postural stability), and 31 (body bradykinesia and hypokinesia) (10). The quality of sleep was also assessed using the Pittsburgh sleep quality index (PSQI), and the quality of life using the Parkinson's disease 39-items questionnaire (PDQ-39), following the methods described in previous studies (11).

Follow-up. Patients were examined at 12 (midpoint), 24 (end of treatment) and 48 weeks (follow-up evaluation). The entire baseline assessment procedure was repeated. The same evaluator who evaluated the baseline indicators performed all subsequent off-medication evaluations and recorded the current medications and adverse events at each visit.

Randomization and blinding. As patients were assigned, the statistician informed the assigned treatment and study coordinator that randomization had occurred. Only the statistician and the coordinator who recruited the patients knew the treatment assignment, but none of these individuals had a role in data collection. The research personnel involved in data collection were blinded to the treatment assignments. The patients knew their treatment assignment, but did not know the study hypothesis.

Statistical analysis. The qualitative information in the baseline data was analyzed using the $\chi^{2}$ test and the quantitative data were analyzed using the independent-samples t-test. The primary outcome measures were the change in the off-medication non-tremor score, tremor score, PSQI and PDQ-39 scores from baseline to 12,24 and 48 weeks. To estimate the treatment effects, comparisons between groups were performed using the Mann-Whitney U-test for two sets of independent samples, as the data did not follow a normal distribution. Statistical analyses were performed using SPSS 17.0 software (SPSS, Inc., Chicago, IL, USA). All statistical tests were two-sided $\mathrm{P}<0.05$ was considered to indicate a statistically significant difference.

\section{Results}

Patients. Between September 2014 and December 2016, 173 patients with PD were screened for study participation and 


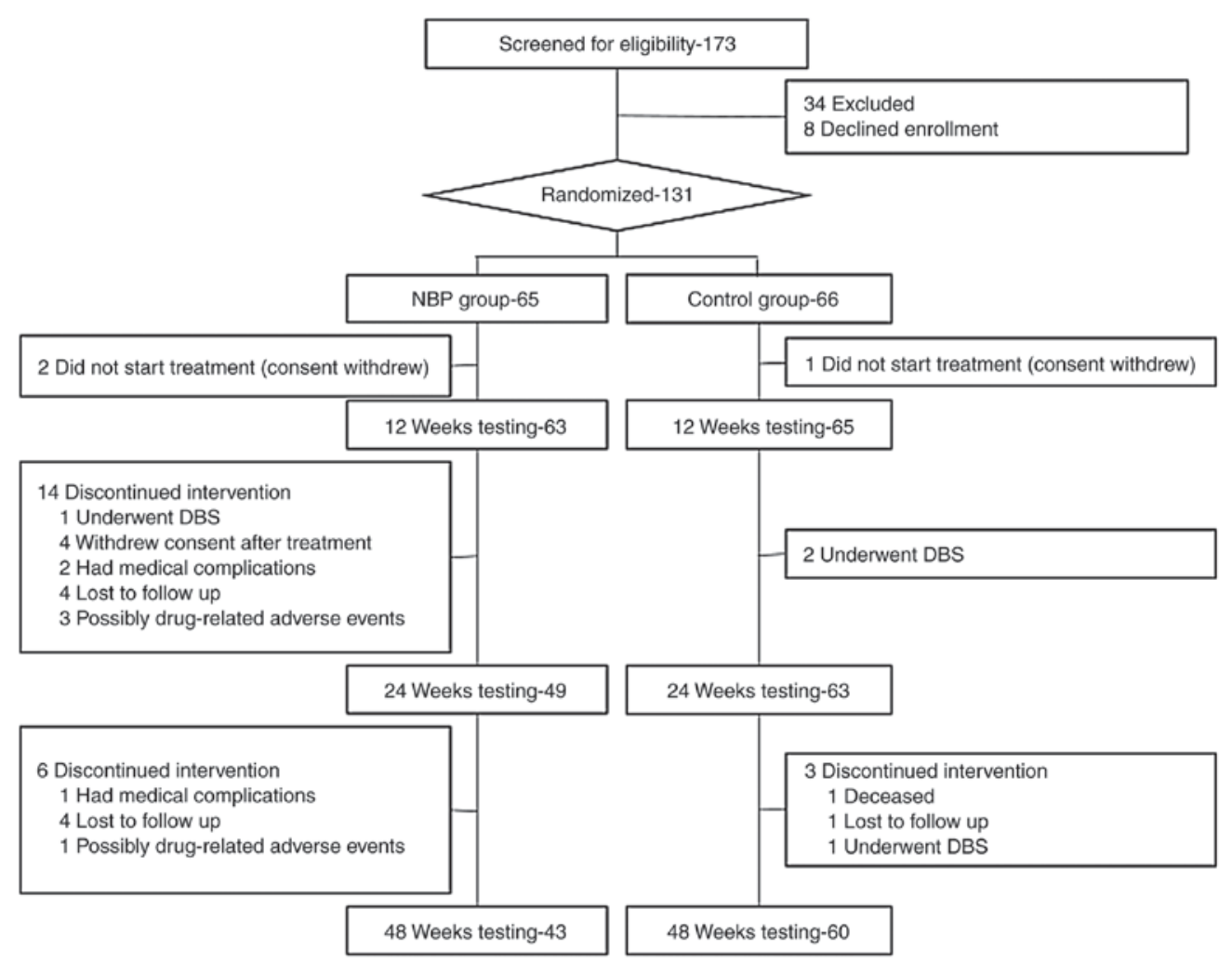

Figure 2. Schematic illustrating the movement of patients through the trial period. DBS, deep brain stimulation; NPB, DL-3-n-butylphthalide.

131 patients were randomly assigned to the NBP group or the control group. A total of 103 patients were followed up until the end of the study, including 43 patients in the NBP group and 60 patients in the control group (Fig. 2). The baseline characteristics were not statistically different between the study groups (Table I). The clinical profiles of the enrolled patients were consistent with the diagnosis of PD. Most enrolled patients were taking concomitant medications, with the most common one being the dopamine receptor agonist $\mathrm{L}$-dopamine, but there were no significant differences in medication usage between the treatment group and the control group.

Motor signs and medication status. The mean off-medication, bradykinesia plus rigidity score and tremor score decreased from baseline at 12,24 and 48 weeks for the patients in the NBP treatment group, The mean off-medication tremor score decreased slightly at 12 and 24 weeks, but increased at 48 weeks. The change from the baseline in the bradykinesia plus rigidity and tremor scores were statistically significant when compared with the control group. (Table II; Fig. 3A and B).

Sleep quality and medication status. The changes in the mean PSQI score for the NBP-treatment group from baseline to 12 and 24 weeks were all statistically significant compared with those in the control group. The scores remained constant at 48 weeks (Table II; Fig. 3C). It was indicated that NBP had a beneficial effect on the sleep quality of PD patients. During the first six months of the study, two patients in the NBP group and 15 patients in the control group required an increase in medication, however, this study did not add other drugs according to the experimental design.
Quality of life. The mean PDQ-39 score decreased from baseline for the NBP treatment group at 12 and 24 weeks, and remained constant at 48 weeks. The difference in the PDQ-39 score changes in the NBP group from the control group was significant (Table II; Fig. 3D). It was therefore indicated that NBP had a beneficial effect on the quality of life of PD patients.

Adverse events. Only three adverse events directly associated with the treatment were noted in the NBP group: NBP allergy characterized by itching and skin rash one week after treatment (1 patient, drug rash improved after discontinuing), slight elevation of ALT 24 weeks after treatment (1 patient, ALT returned to normal 1 week after stopping the drug) and a mild gastrointestinal reaction (2 patients, reactions improved 30 min after taking the medicine).

\section{Discussion}

The pathogenesis of PD includes synaptic transmission, the cytoplasmic cycle, lysosomal autophagy and multiple molecular mechanisms, including mitochondrial metabolism. Thus far, no effective medication has been developed to interfere with this specific pathogenesis. Clinical trials, including those that evaluated adeno-associated virus serotype 2-nerve growth factor, coenzyme Q10, creatine, pramipexole and pioglitazone, all reported negative results $(12,13)$. As the first randomized, controlled, blinded clinical trial of NBP for PD, the present study should be considered exploratory. The methods and results of the present study may provide an important reference for patient selection, outcome measures and study duration of future PD drug trials. Of note, the results 
Table I. Characteristics of patients at baseline, by treatment group.

\begin{tabular}{|c|c|c|c|c|}
\hline Characteristic & $\operatorname{NBP}(n=43)$ & Control $(n=60)$ & $\chi^{2} / \mathrm{t}$ & P-value \\
\hline Age (years) & $60.00 \pm 9.82$ & $61.92 \pm 9.77$ & -0.980 & 0.330 \\
\hline Duration of disease (months) & $38.30 \pm 26.84$ & $48.08 \pm 31.36$ & -1.655 & 0.101 \\
\hline \multicolumn{5}{|l|}{ UPDRS, off } \\
\hline \multicolumn{5}{|l|}{ UPDRS III, off } \\
\hline medication & $30.72 \pm 14.87$ & $34.42 \pm 17.20$ & -1.164 & 0.247 \\
\hline MMSE & $26.51 \pm 5.84$ & $24.53 \pm 6.90$ & 1.572 & 0.119 \\
\hline ESS & $5.37 \pm 4.81$ & $5.52 \pm 5.30$ & -0.142 & 0.887 \\
\hline PSQI & $8.63 \pm 6.94$ & $6.72 \pm 4.18$ & 1.608 & 0.113 \\
\hline FSS & $3.28 \pm 2.02$ & $3.73 \pm 2.12$ & -1.093 & 0.277 \\
\hline HRSD & $14.16 \pm 8.87$ & $15.43 \pm 8.28$ & -0.745 & 0.458 \\
\hline HAMA & $8.16 \pm 5.91$ & $9.02 \pm 5.50$ & -0.753 & 0.453 \\
\hline $\begin{array}{l}\text { PDQ-39, off } \\
\text { medication }\end{array}$ & $40.49 \pm 27.80$ & $48.37 \pm 29.41$ & -1.372 & 0.173 \\
\hline Gender & & & 0.434 & 0.510 \\
\hline Male & $23(53.5)$ & $36(60.0)$ & & \\
\hline Female & $20(46.5)$ & $24(40.0)$ & & \\
\hline Levodopa equivalent dose & & & 1.131 & 0.288 \\
\hline No & $18(41.9)$ & $19(31.7)$ & & \\
\hline Yes & $25(58.1)$ & $41(68.3)$ & & \\
\hline H-Y stage, off medication & & & 5.072 & 0.407 \\
\hline 1 & $12(27.9)$ & $14(23.3)$ & & \\
\hline 1.5 & $10(23.3)$ & $12(20.0)$ & & \\
\hline 2 & $6(14.0)$ & $5(8.3)$ & & \\
\hline 3 & $6(14.0)$ & $17(28.3)$ & & \\
\hline 4 & $9(20.9)$ & $10(16.7)$ & & \\
\hline 5 & $0(0.0)$ & $2(3.3)$ & & \\
\hline
\end{tabular}

Values are expressed as $\mathrm{n}(\%)$ or the mean \pm standard deviation. NBP, DL-3-n-butylphthalide; UPDRS, Unified Parkinson's Disease Rating Scale; MMSE, Mini Mental State Examination; ESS, Epworth sleep rating scale; PSQI, Pittsburgh sleep quality index; FSS, Fatigue Severity Scale; HRSD, Hamilton depression scale; HAMA, Hamilton anxiety scale; PDQ-39, Parkinson's disease quality of life 39-items questionnaire.

of the present study indicated that NBP may be a promising treatment option for PD.

The end-points of the present study were off-medication tremor score and non-tremor (bradykinesia plus rigidity) score based on the predominant motor features in the UPDRS III motor examination section score, rather than generally assessing the patients' motor symptoms using the UPDRS III scores.

According to previous reports, $50 \%$ of patients with PD complained of slowness of movement, including the initiation and execution of movements, particularly sequential and volitional actions, and $40 \%$ of patients are likely to complain of tremulousness of the hand at rest (14). Based on the different abovementioned clinical symptoms, patients with PD were subtyped into one of two clinical groups, non-tremor-dominant subtype (ART) and tremor-dominant subtype (TDT), and evaluated using the non-tremor score and tremor score (15).

ART and TDT have different clinical signs, markers and pathogeneses. In ART, PD progresses more rapidly (16), and in the early stage, the dopaminergic system is more severely impaired (17). Patients with the ART of PD have consistently been reported to exhibit a broader array of NMS than TDT, involving earlier autonomic features and later cognitive impairment (1). Furthermore, previous studies have indicated that the disease in NMS-dominant phenotypes may begin peripherally in the gastrointestinal tract (18) and link with the heterogeneity of the initiating neuropathology to reflect the convergence of deficits in multiple transmitter systems and pathways, including the cholinergic, serotonergic and noradrenergic systems, occurring in PD (19).

The present results demonstrated a favorable effect of NBP in the treatment of patients with bradykinesia plus rigidity symptoms. The efficacy of NBP in PD may be mediated by multiple targets involved in the pathogenesis of this disorder. Data from animal and cell models suggest that NBP protects dopaminergic neurons by blocking the upregulation of intracellular reactive oxygen species production, inhibiting a decrease in cleaved caspase-3/caspase-3 and B-cell lymphoma 2 (Bcl-2)/Bcl-2-associated $\mathrm{X}$ protein ratios (20), scavenging free radicals (21), increasing the number of synapses and apical 
Table II. Bradykinesia plus rigidity, tremor, sleep quality and quality of life at each follow-up visit by treatment group.

\begin{tabular}{|c|c|c|c|c|c|c|}
\hline \multirow[b]{2}{*}{ Time-point } & \multicolumn{2}{|c|}{ Score at follow-up visit } & \multicolumn{2}{|c|}{ Change from baseline ${ }^{a}$} & \multirow[b]{2}{*}{ Z-value } & \multirow[b]{2}{*}{ P-valu } \\
\hline & Control $(n=60)$ & $\operatorname{NBP}(n=43)$ & Control $(n=60)$ & NBP $(n=43)$ & & \\
\hline \multicolumn{7}{|c|}{$\begin{array}{l}\text { Bradykinesia plus rigidity score, } \\
\text { off medication }\end{array}$} \\
\hline Baseline & $33.05 \pm 17.57$ & $28.86 \pm 15.85$ & & & & \\
\hline 12 weeks & $33.33 \pm 18.24$ & $24.26 \pm 13.57$ & $0.28 \pm 6.21$ & $-4.60 \pm 3.51$ & -7.562 & $<0.001$ \\
\hline 24 weeks & $33.55 \pm 18.02$ & $21.72 \pm 11.71$ & $0.50 \pm 9.48$ & $-7.14 \pm 5.81$ & -7.625 & $<0.001$ \\
\hline 48 weeks & $36.18 \pm 19.38$ & $21.74 \pm 11.77$ & $3.13 \pm 11.28$ & $-7.12 \pm 5.96$ & -7.611 & $<0.001$ \\
\hline \multicolumn{7}{|c|}{ Tremor score, off medication } \\
\hline Baseline & $48.10 \pm 29.22$ & $40.49 \pm 27.80$ & & & & \\
\hline 12 weeks & $48.43 \pm 28.88$ & $31.42 \pm 22.66$ & $0.33 \pm 9.54$ & $-9.07 \pm 8.82$ & -8.432 & $<0.001$ \\
\hline 24 weeks & $48.08 \pm 28.07$ & $27.23 \pm 18.15$ & $-0.02 \pm 12.26$ & $-13.26 \pm 11.52$ & -8.022 & $<0.001$ \\
\hline 48 weeks & $50.55 \pm 28.61$ & $27.33 \pm 18.18$ & $2.45 \pm 13.52$ & $-13.16 \pm 11.53$ & -7.963 & $<0.001$ \\
\hline
\end{tabular}

Pittsburgh sleep quality index

scores, off medication

$\begin{array}{lrrrrrr}\text { Baseline } & 15.43 \pm 8.28 & 14.16 \pm 8.87 & & & & \\ 12 \text { weeks } & 15.43 \pm 8.28 & 9.93 \pm 5.94 & 0.00 \pm 2.82 & -3.47 \pm 3.99 & -3.945 & <0.001 \\ 24 \text { weeks } & 15.60 \pm 8.15 & 9.70 \pm 5.78 & 1.88 \pm 4.22 & -3.93 \pm 4.48 & -5.361 & <0.001 \\ 48 \text { weeks } & 15.60 \pm 8.15 & 9.70 \pm 5.78 & 1.88 \pm 4.22 & -3.93 \pm 4.48 & -5.361 & <0.001\end{array}$

Parkinson's disease 39-items questionnaire scores, off medication

\begin{tabular}{lcclccc} 
Baseline & $48.10 \pm 29.22$ & $40.49 \pm 27.80$ & & & & \\
12 weeks & $48.43 \pm 28.88$ & $31.42 \pm 22.66$ & $0.33 \pm 9.54$ & $-9.07 \pm 8.82$ & -8.432 & $<0.001$ \\
24 weeks & $48.08 \pm 28.07$ & $27.23 \pm 18.15$ & $0.02 \pm 12.26$ & $-13.26 \pm 11.52$ & -8.022 & $<0.001$ \\
48 weeks & $50.55 \pm 28.61$ & $27.33 \pm 18.18$ & $2.45 \pm 13.52$ & $-13.16 \pm 11.53$ & -7.963 & $<0.001$ \\
\hline
\end{tabular}

${ }^{a}$ Negative change scores indicate improvement and positive change scores indicate worsening compared with the baseline. ${ }^{\mathrm{b}} \mathrm{Change}$ from baseline in NBP treatment group vs. control group. NBP, DL-3-n-butylphthalide.

A
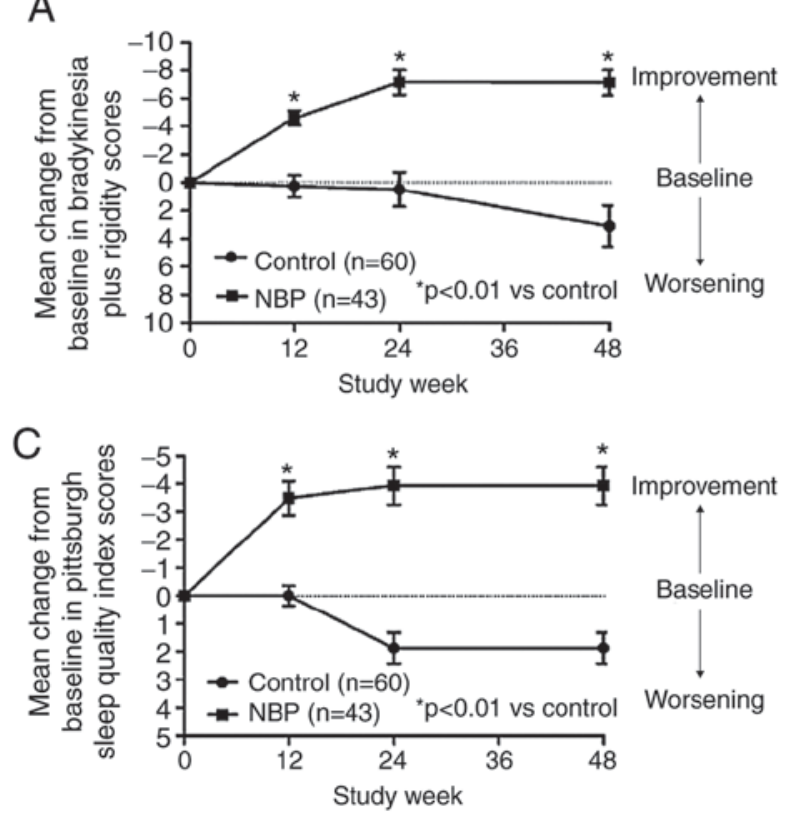
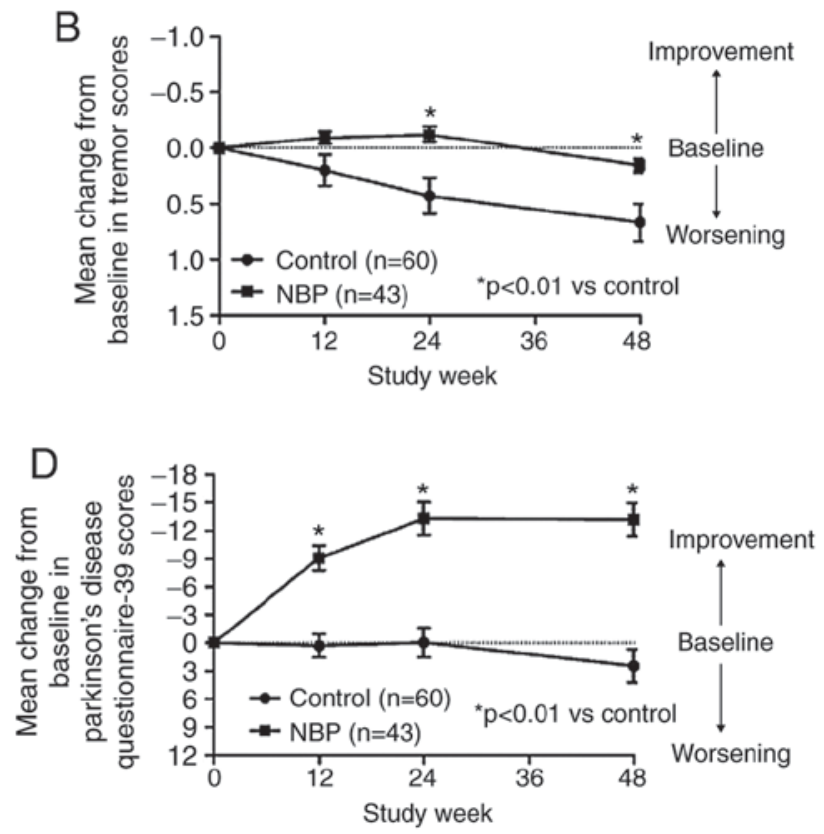

Figure 3. Change from baseline scores for all outcomes in the two groups. Change from baseline in (A) the Bradykinesia plus rigidity scores, (B) tremor scores, (C) Pittsburgh sleep quality index scores and (D) Parkinson's disease 39-items questionnaire scores, at 12,24 and 48 weeks. Negative change scores indicate improvement and positive change scores indicate worsening in the respective scores. Values are expressed as the mean \pm standard error of the mean. $\mathrm{P}<0.01$ vs. control group. NPB, DL-3-n-butylphthalide. 
dendritic thorns, attenuating neural inflammatory responses by reducing oxidative stress, decreasing nuclear fragmentation, retaining the mitochondrial membrane potential (7), and activating autophagy-mediated $\alpha$-synuclein degradation (22).

Previous animal experiments performed by our group indicated that NBP reduces microglial activation in mice with LPS-induced PD. LPS is closely associated to the pathogenesis of PD $(23,24)$ and is derived from the components of Gram-negative bacterial cells, which are common in the intestinal flora. The intestinal flora is closely linked to the immune system, including the nervous system (25), and intestinal flora imbalances are associated with PD $(23,26)$. A dysregulation of the intestinal flora and intestinal inflammation lead to increased intestinal mucosal permeability, resulting in LPS entering the body and causing neuroinflammation. During this process, the intestinal nerve is the first to be affected and constipation and other non-motor symptoms appear at the early stages. As these lesions move along the vagus nerve, the brain stem, midbrain and cerebral cortex are involved, leading to sleep disorders, symptoms of mobility disorders and cognitive impairment $(23,26,27)$. The clinical manifestations for the PD subtype of this pathogenesis are NMS at the early stage and primarily non-tremor (bradykinesia plus rigidity) movement symptoms at the later stage (1). As expected, the results of the present study indicated that NBP mainly improved the bradykinesia plus rigidity symptom in patients with PD. Therefore, it is likely that the mechanism of NBP in PD may be the attenuation of neural inflammatory responses, which elicits a neuroprotective effect and improves and prevents PD development.

The present results demonstrated a favorable effect of NBP in the treatment of PD patients with sleep disorders. Sleep disorders are a common NMS of PD that occurs due to lesions located in the medulla oblongata in the brain stem. There are two possible mechanisms by which NBP may improve sleep disorders in patients with PD. First, similar to how NBP improves the bradykinesia plus rigidity symptom of PD, NBP may exert neuroprotective effects through attenuation of neuroinflammatory responses. Furthermore, NBP was first purified from the seeds of Apium graveolens (Water celery) in 1978 at the Institute of Medicine of the Chinese Academy of Medical Sciences, inspired by a folk prescription for 'boiling of sailboat canvas and celery seeds together in water for the treatment of epilepsy', and in 1980, the compound was synthesized at the same institute for the first time (28). The pharmacological tests at that time demonstrated that NBP exhibited significant effects on the CNS, including anti-convulsant and sedative actions. This sedative effect may be one of the mechanisms by which NBP improves sleep disorders in PD.

Of note, the present study have several limitations. First, it was not a double-blinded trial, as it was not possible to perform blinding with respect to regular medication and compliance with the follow-up questionnaire. Furthermore, the sample size was small. Finally, the control group was not given a placebo drug. However, these issues were minor, as the participants did not know the group assignment until baseline testing was completed and the evaluators did not know it either until the study was completed.

In conclusion, the present preliminary study suggested that 6 months of NBP treatment was effective for improving bradykinesia plus rigidity and sleep quality in patients with $\mathrm{PD}$, providing a promising option for early intervention in this disease. Future experiments with larger sample sizes and a longer duration are required to further test the efficacy of NBP in PD and its different subtypes.

\section{Acknowledgements}

Not applicable.

\section{Funding}

The present study was supported by the National Natural Science Foundation of China province in 2016 (grant no. 81641050) and The Strategic Priority Research Program (Pilot study) 'Biological basis of aging and intervention strategies' of the Chinese Academy of Sciences (grant no. XDPB10).

\section{Availability of data and materials}

The datasets used and/or analysed during the present study are available from the corresponding author on reasonable request.

\section{Authors' contributions}

$\mathrm{HZ}$ was responsible for drafting manuscript, critically revising it for important knowledge content, participating in the experimental design, and agreeing to be responsible for all aspects of the work to ensure proper investigation and resolution of issues related to the accuracy or completeness of any part of the work. MY analyzed baseline data for PD patients. WX acquired the data. MY analyzed and interpreted the data. XL was involved in XL assessing the patients for fullfilling the inclusion criteria, and revising the manuscript critically for important intellectual content. YC designed the experiments, drafted the manuscript, and critically modified important content. All authors read and approved the final version of the manuscript.

\section{Ethical approval and consent to participate}

The present study received full approval from the First Affiliated Hospital of Bengbu Medical College Institutional Review Board (Bengbu, China). Informed consent was obtained from each participant.

\section{Patient consent for publication}

Not applicable.

\section{Competing interests}

The authors declare that they have no competing interests.

\section{References}

1. Marras $\mathrm{C}$ and Chaudhuri KR: Nonmotor features of Parkinson's disease subtypes. Mov Disord 31: 1095-1102, 2016.

2. Liu CL, Liao SJ, Zeng JS, Lin JW, Li CX, Xie LC, Shi XG and Huang RX: dl-3n-butylphthalide prevents stroke via improvement of cerebral microvessels in RHRSP. J Neurol Sci 260: 106-113, 2007. 
3. Zhao Y, Lee JH, Chen D, Gu X, Caslin A, Li J, Yu SP and Wei L: DL-3-n-butylphthalide induced neuroprotection, regenerative repair, functional recovery and psychological benefits following traumatic brain injury in mice. Neurochem Int 111: 82-92, 2017.

4. Jia J, Wei C, Liang J, Zhou A, Zuo X, Song H, Wu L, Chen X, Chen S, Zhang J, et al: The effects of DL-3-n-butylphthalide in patients with vascular cognitive impairment without dementia caused by subcortical ischemic small vessel disease: A multicentre, randomized, double-blind, placebo-controlled trial. Alzheimers Dement 12: 89-99, 2016.

5. Cui LY, Zhu YC, Gao S, Wang JM, Peng B, Ni J, Zhou LX, He J and Ma XQ: Ninety-day administration of dl-3-n-butylphthalide for acute ischemic stroke: A randomized, double-blind trial. Chin Med J (Engl) 126: 3405-3410, 2013.

6. Huang JZ, Chen YZ, Su M, Zheng HF, Yang YP, Chen J and Liu CF: dl-3-n-Butylphthalide prevents oxidative damage and reduces mitochondrial dysfunction in an MPP(+)-induced cellular model of Parkinson's disease. Neurosc Lett 475: 89-94, 2010.

7. Xiong N, Huang J, Chen C, Zhao Y, Zhang Z, Jia M, Zhang Z, Hou L, Yang H, Cao X, et al: Dl-3-n-butylphthalide, a natura antioxidant, protects dopamine neurons in rotenone models for Parkinson's disease. Neurobiol Aging 33: 1777-1791, 2012.

8. Hughes AJ, Daniel SE, Kilford L and Lees AJ: Accuracy of clinical diagnosis of idiopathic Parkinson's disease: A clinico-pathological study of 100 cases. J Neurol Neurosurg Psychiatry 55: 181-184, 1992

9. Corcos DM, Robichaud JA, David FJ, Leurgans SE Vaillancourt DE, Poon C, Rafferty MR, Kohrt WM and Comella CL: A two-year randomized controlled trial of progressive resistance exercise for Parkinson's disease. Mov Disord 28 1230-1240, 2013.

10. Schiess MC, Zheng H, Soukup VM, Bonnen JG and Nauta HJ: Parkinson's disease subtypes: Clinical classification and ventricular cerebrospinal fluid analysis. Parkinsonism Relat Disord 6 : 69-76, 2000.

11. Thomas S, Reading J and Shephard RJ: Revision of the physical activity readiness questionnaire (PAR-Q). Can J Sport Sci 17: 338-345, 1992

12. Volta M, Milnerwood AJ and Farrer MJ: Insights from late-onset familial parkinsonism on the pathogenesis of idiopathic Parkinson's disease. Lancet Neurol 14: 1054-1064, 2015.

13. Calabresi P and Di Filippo M: Multitarget disease-modifying therapy in Parkinson's disease? Lancet Neurol 14: 975-976, 2015.

14. Spiegel J, Möllers MO, Jost WH, Fuss G, Samnick S, Dillmann U, Becker G and Kirsch CM: FP-CIT and MIBG scintigraphy in early Parkinson's disease. Mov Disord 20: 552-561, 2005

15. Ahlskog JE: Diagnosis and differential diagnosis of Parkinson's disease and parkinsonism. Parkinsonism Relat Disord 7: 63-70, 2000.

16. Chiaravalloti A, Stefani A, Tavolozza M, Pierantozzi M, Di Biagio D, Olivola E, Di Pietro B, Stampanoni M, Danieli R, Simonetti G, et al: Different patterns of cardiac sympathetic denervation in tremor-type compared to akinetic-rigid-type Parkinson's disease: Molecular imaging with ${ }^{123}$ I-MIBG. Mol Med Rep 6: 1337-1342, 2012.
17. Schillaci O, Chiaravalloti A, Pierantozzi M, Di Pietro B, Koch G, Bruni C, Stanzione P and Stefani A: Different patterns of nigrostriatal degeneration in tremor type versus the akinetic-rigid and mixed types of Parkinson's disease at the early stages: Molecular imaging with 123I-FP-CIT SPECT. Int J Mol Med 28: 881-886, 2011.

18. Shannon KM, Keshavarzian A, Dodiya HB, Jakate S and Kordower JH: Is alpha-synuclein in the colon a biomarker for premotor Parkinson's disease? Evidence from 3 cases. Mov Disord 27: 716-719, 2012.

19. Espay AJ, LeWitt PA and Kaufmann H: Norepinephrine deficiency in Parkinson's disease: The case for noradrenergic enhancement. Mov Disord 29: 1710-1719, 2014

20. Zhao J,Liu J, XuE,Liu Y,Xie A and Xiong H: dl-3-n-Butylphthalide attenuation of methamphetamine-induced neurotoxicity in SH-SY5Y neuroblastoma cells. Life Sci 165: 16-20, 2016.

21. Zhou J, Zhang YH, Song HZ, Ji H, Wang XL, Wang L, Qian J, Ling JJ and Ping FF: 5d, a novel analogue of 3-n-butylphthalide, decreases NADPH oxidase activity through the positive regulation of CK2 after ischemia/reperfusion injury. Oncotarget 7: 39444-39457, 2016.

22. Liu K, Huang J, Chen R, Zhang T, Shen L, Yang J and Sun X: Protection against neurotoxicity by an autophagic mechanism. Braz J Med Biol Res 45: 401-407, 2012.

23. Qin L, Wu X, Block ML, Liu Y, Breese GR, Hong JS, Knapp DJ and Crews FT: Systemic LPS causes chronic neuroinflammation and progressive neurodegeneration. Glia 55: 453-462, 2007.

24. Pal GD, Shaikh M, Forsyth CB, Ouyang B, Keshavarzian A and Shannon KM: Abnormal lipopolysaccharide binding protein as marker of gastrointestinal inflammation in Parkinson disease. Front Neurosci 9: 306, 2015.

25. Hand TW, Vujkovic-Cvijin I, Ridaura VK and Belkaid Y: Linking the microbiota, chronic disease, and the immune system. Trends Endocrinol Metab 27: 831-843, 2016.

26. Sampson TR, Debelius JW, Thron T, Janssen S, Shastri GG, Ilhan ZE, Challis C, Schretter CE, Rocha S, Gradinaru V, et al: Gut microbiota regulate motor deficits and neuroinflammation in a model of Parkinson's disease. Cell 167: 1469-1480.e12, 2016.

27. McCann H, Cartwright H and Halliday GM: Neuropathology of $\alpha$-synuclein propagation and braak hypothesis. Mov Disord 31: 152-160, 2016.

28. Yang Q, Hu YD, Wang XF and Zheng FS: Dl-3n-butylphthalide reduces epileptiform activity through GluA2-lacking calcium-permeable AMPARs in epilepsy models. Oncotarget 8: 98242-98257, 2017. 